\title{
General Psychiatry Adjunctive Peony-Glycyrrhiza decoction for antipsychotic-induced hyperprolactinaemia: a meta-analysis of randomised controlled trials
}

\author{
Wei Zheng, ${ }^{1}$ Dong-Bin Cai, ${ }^{2}$ Hai-Yan Li, ${ }^{1}$ Yu-Jie Wu, ${ }^{1}$ Chee H Ng, ${ }^{3}$ \\ Gabor S Ungvari, ${ }^{4,5}$ Shan-Shan Xie, ${ }^{6}$ Zhan-Ming Shi, ${ }^{7}$ Xiao-Min Zhu, ${ }^{8}$ \\ Yu-Ping Ning, ${ }^{1}$ Yu-Tao Xiang ${ }^{9}$
}

To cite: Zheng W, Cai D-B, Li H-Y, et al. Adjunctive Peony-Glycyrrhiza decoction for antipsychotic-induced hyperprolactinaemia: a meta-analysis of randomised controlled trials. General Psychiatry 2018;31:e100003. doi:10.1136/ gpsych-2018-100003

WZ and D-BC contributed equally.

Received 13 August 2018 Accepted 13 August 2018

Check for updates

(C) Author(s) (or their employer(s)) 2018. Re-use permitted under CC BY-NC. No commercial re-use. See rights and permissions. Published by BMJ.

For numbered affiliations see end of article.

Correspondence to Dr Yu-Tao Xiang;

xyutly@gmail.com

\section{ABSTRACT}

Background Hyperprolactinaemia is a common adverse effect of antipsychotics (APs). The results of PeonyGlycyrrhiza decoction (PGD) as a potentially useful adjunctive treatment for hyperprolactinaemia are inconsistent. Aim This meta-analysis of randomised controlled trials (RCTs) examined the efficacy and safety of adjunctive PGD therapy for AP-induced hyperprolactinaemia.

Methods English (PubMed, Embase, Cochrane Library, PsycINF0) and Chinese (Chinese National Knowledge Infrastructure, Wanfang Data) databases were systematically searched up to 10 June 2018. The inclusion criteria were based on PICOS—Participants: adult patients with schizophrenia; Itervention: PGD plus APs; Comparison: APs plus placebo or AP monotherapy; Outcomes: efficacy and safety; Study design: RCTs. The weighted mean difference (WMD) and risk ratio (RR) along with their 95\% Cls were calculated using Review Manager (RevMan) V.5.3 software.

Results Five RCTs ( $n=450)$ were included and analysed. Two RCTs ( $n=140$ ) were double-blind and four RCTs $(\mathrm{n}=409)$ reported 'random' assignment with specific description. The PGD group showed a significantly lower serum prolactin level at endpoint than the control group ( $\mathrm{n}=380$, WMD: $-32.69 \mathrm{ng} / \mathrm{mL}$ (95\% Cl -41.66 to 23.72 ), $\left.p<0.00001, P^{2}=97 \%\right)$. Similarly, the superiority of PGD over the control groups was also found in the improvement of hyperprolactinaemia-related symptoms. No difference was found in the improvement of psychiatric symptoms assessed by the Positive and Negative Syndrome Scale $(\mathrm{n}=403$, WMD: -0.62 ( $95 \% \mathrm{Cl}-2.38$ to 1.15$), p=0.49, \stackrel{P}{P}=0 \%$ ). There were similar rates of all-cause discontinuation $(n=330, R R$ 0.93 (95\% $\mathrm{Cl} 0.63$ to 1.37 ), $p=0.71, P=0 \%$ ) and adverse drug reactions between the two groups. According to the Grading of Recommendations Assessment, Development and Evaluation approach, the level of evidence of primary and secondary outcomes ranged from 'very low' (14.3\%), 'Iow' (42.8\%), 'moderate' (14.3\%), to 'high' (28.6\%).

Conclusions Current evidence supports the adjunctive use of PGD to suppress elevated prolactin and improve prolactininduced symptoms without significant adverse events in adult patients with AP-induced hyperprolactinaemia. Highquality RCTs with longer duration are needed to confirm these findings.

Trial registration number 42016037017 .

\section{INTRODUCTION}

Hyperprolactinaemia induced by antipsychotics (APs), defined as an abnormally high serum prolactin level of greater than $25 \mathrm{ng} / \mathrm{mL}$ for men and $20 \mathrm{ng} / \mathrm{mL}$ for women, ${ }^{12}$ is a common adverse drug reaction (ADR). ${ }^{3}$ APs induce hyperprolactinaemia by enhancing prolactin secretion through their dopamine-blocking action in the tuberoinfundibular system. ${ }^{4}$ The prevalence of hyperprolactinaemia is up to $76 \%-86 \%$ in patients receiving AP treatment. ${ }^{5}$ Hyperprolactinaemia could result in severe menstrual irregularities, including amenorrhoea, gynaecomastia, galactorrhoea, sexual dysfunction, infertility and decreased bone mineral density, all of which would contribute to poor treatment adherence and reduced quality of life. ${ }^{67}$

Several treatment strategies have been suggested to suppress hyperprolactinaemia and improve the associated symptoms, although some of these strategies remain controversial. The frequently used treatment options include (1) dopamine agonists, such as bromocriptine (BMT), amantadine and cabergoline, all of which could induce abnormal involuntary movements; ${ }^{18}$ (2) metformin, which may cause gastrointestinal symptoms; ${ }^{9}$ (3) aripiprazole, which may cause sedation, insomnia and headache $;^{10-12}$ (4) using the lowest possible therapeutic dose of AP; however, this strategy may increase the risk of relapse; ${ }^{13}$ and (5) switching to another AP, risking the occurrence of other ADRs, such as metabolic syndrome and sedation. $^{14} 15$

In the past two decades growing evidence has suggested that Peony-Glycyrrhiza decoction (PGD), an herbal medicine formula consisting of Paeonia and Glycyrrhiza radices (shaoyao-gancao-tang in Chinese and shakuyaku-kanzo-toin in Japanese, TJ-68), 
Search terms (in the title or abstract): ("Peony-Glycyrrhiza" [MeSH] OR paeonia lacliflora and Glycyrrhiza uralensis OR shakuyaku-kanzo-to OR shaoyao gancao OR glycyrrhiza OR paeoniae) AND ("prolactin"[MeSH] OR hyperprolactinemia OR prolactin OR amenorrhea OR menstrual irregularities OR oligomenorrhea OR galactorrhea OR gynecomastia)

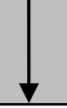

A total of 241 articles published by June 10, 2018 were identified from PubMed $(n=26)$, Embase $(n=91)$, PsycINFO $(n=7)$, and Cochrane Library $(n=18)$, China Knowledge Network $(n=32)$, and WanFang Database $(n=67)$

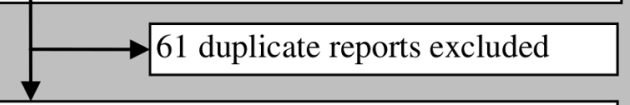

The titles or abstract of the 180 articles were screened

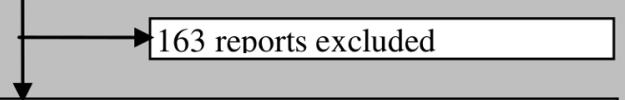

The full text of 17 articles were examined to determine whether or not they reported the efficacy and safety of adjunctive PGD for antipsychotic-induced Hyperprolactinaemia

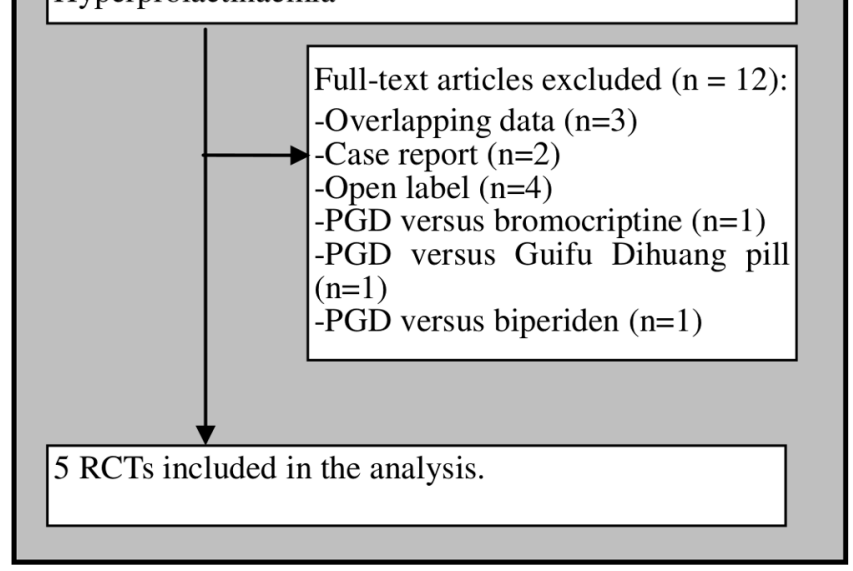

Figure 1 Flow chart of identification of studies. PGD, Peony-Glycyrrhizadecoction; RCT, randomized controlled trial.

can significantly improve prolactin-induced symptoms in patients with AP-induced hyperprolactinaemia. ${ }^{16-22}$ A number of case reports, ${ }^{21} 22$ observational studies ${ }^{17-20}$ and randomised controlled trials (RCTs) ${ }^{1623-26}$ of adjunctive PGD for AP-induced hyperprolactinaemia have been published with mixed results.

This meta-analysis set out to assess the efficacy and safety of adjunctive PGD in the treatment of AP-induced hyperprolactinaemia, including recent studies published in Chinese-language and English-language journals.

\section{METHODS}

\section{Inclusion and exclusion criteria}

Two independent investigators (WZ, D-BC) assessed and screened the eligibility of studies based on the following criteria of PICOS: Participants: adult patients with schizophrenia; Intervention: PGD plus APs; Comparison: APs plus placebo or AP monotherapy; Outcomes: the primary outcome measures were efficacy of treatment (serum prolactin level and prolactin-related symptoms: reduction in the severity of galactorrhoea, amenorrhoea and oligomenorrhoea) - key secondary outcomes were improvement of psychiatric symptoms assessed by the Positive and Negative Syndrome Scale (PANSS) ${ }^{27}$ or the Brief Psychiatric Rating Scale, ${ }^{28}$ ADRs assessed using the Treatment Emergent Symptom Scale (TESS), ${ }^{29}$ and all-cause discontinuation; Study design: only RCTs examining the efficacy and safety of adjunctive PGD for AP-induced hyperprolactinaemia. Case report/series, observational studies and meta-analyses and systematic reviews were excluded.

\section{Search strategy}

Two reviewers (WZ, D-BC) independently searched PubMed, Embase, Cochrane Library, PsycINFO, Chinese National Knowledge Infrastructure and Wanfang Data for trials evaluating adjunctive PGD for AP-induced hyperprolactinaemia from inception of these databases until 10 June 2018. The keywords used for the searches included the following: ("Peony-Glycyrrhiza" (MeSH) OR paeonia lacliflora and Glycyrrhiza uralensis OR shakuyaku-kanzo-to OR shaoyao gancao OR glycyrrhiza OR paeoniae) AND ("prolactin" (MeSH) OR hyperprolactinemia OR prolactin OR amenorrhea OR menstrual irregularities OR oligomenorrhea OR galactorrhea OR gynecomastia). Reference lists from relevant review articles for additional studies were hand-searched. The two reviewers (WZ, D-BC) independently assessed all trials by judging their clinical, methodological and statistical heterogeneity to decide which ones to include in the qualitative or quantitative synthesis. Any disagreement was discussed with a third reviewer (Y-TX).

\section{Data extraction}

Data of each study were independently identified, checked and extracted by the two reviewers (WZ, D-BC). Results based on intention-to-treat were preferred to observed cases data. Inconsistencies were resolved by consensus involving a third reviewer. In addition, missing information was obtained by contacting the first or corresponding authors, or was extracted from graphs or figures of included RCTs if possible.

\section{Evaluation of quality of included studies}

The included RCTs were assessed by two reviewers (WZ, D-BC) using the Cochrane risk of bias with seven dimensions as follows: (1) random sequence generation; (2) allocation concealment; (3) blinding of the subjects and the treatment providers; (4) blinding of the result evaluators; (5) incomplete results data; (6) selective reporting; and (7) other potential risks. ${ }^{30}$ The quality of evidence and strength of recommendations of outcome measure of PGD for hyperprolactinaemia were assessed using the Grading of Recommendations Assessment, Development 
Table 1 Characteristics of the included studies

\begin{tabular}{|c|c|c|c|c|c|c|c|}
\hline Study & $\begin{array}{l}\text { Duration } \\
\text { (weeks) }\end{array}$ & $\begin{array}{l}\text { Patients, } \mathbf{n} \\
\text { (intervention/control) } \\
\text { Male, } \mathbf{n} \text { (intervention/ } \\
\text { control) }\end{array}$ & $\begin{array}{l}\text { Age } \\
\text { (years) }^{*}\end{array}$ & AP & PGD dose & $\begin{array}{l}\text { Change of } \\
\text { serum prolactin } \\
\text { level }(\mathrm{ng} / \mathrm{mL})\end{array}$ & $\begin{array}{l}\text { Hyperprolactinaemia- } \\
\text { induced adverse } \\
\text { effects at endpoint } †\end{array}$ \\
\hline Xie et al, $2015^{16}$ & 12 & $\begin{array}{l}120(80 / 40) \\
0\end{array}$ & 32 & Risperidone & $\begin{array}{l}\text { A group: } \\
\text { Paeoniae Radix } \\
\text { Alba }(30 \mathrm{~g}) \text { and } \\
\text { Glycyrrhizae } \\
\text { Radix Et } \\
\text { Rhizoma }(30 \mathrm{~g}) \\
\text { B group: } \\
\text { Paeoniae Radix } \\
\text { Alba }(30 \mathrm{~g}) \text { and } \\
\text { Glycyrrhizae } \\
\text { Radix Et } \\
\text { Rhizoma }(15 \mathrm{~g})\end{array}$ & $\begin{array}{l}\text { PGD (A group): } \\
-54.70 \pm 20.91 \\
\text { PGD (B group): } \\
-55.40 \pm 24.62 \\
\text { Control: } \\
9.77 \pm 23.20\end{array}$ & NA \\
\hline Yang et al, $2017^{25}$ & 8 & $\begin{array}{l}41(21 / 20) \\
0\end{array}$ & 28 & Amisulpride & $\begin{array}{l}\text { Paeoniae Radix } \\
\text { Alba ( } 60 \mathrm{mg}) \\
\text { and Glycyrrhizae } \\
\text { Radix Et } \\
\text { Rhizoma (30 mg) }\end{array}$ & $\begin{array}{l}\text { PGD: } \\
-22.62 \pm 4.92 \\
\text { Placebo: } \\
-0.98 \pm 5.35\end{array}$ & $\begin{array}{l}\text { PGDף: } 79 \% \\
\text { Placebo: } 0 \%\end{array}$ \\
\hline
\end{tabular}

*Available data were extracted based on mean baseline value of each included trials.

${ }^{\dagger}$ Percentage of improvement.

‡Including risperidone, paliperidone, sulpiride, amisulpride, lanzapine, ziprasidone, quetiapine, perphenazine, clozapine and chlorpromazine.

\$Paeonia and Glycyrrhiza radices in a ratio of $1: 1$ in weight.

"Including 2 (amenorrhoea), 4 (abnormal menstruation), 3 (galactorrhoea), 4 (reduced libido) and 2 (sexlessness).

${ }^{* *}$ No detailed use of AP.

AP, antipsychotic ; NA, not available; PGD, Peony-Glycyrrhiza decoction.

and Evaluation (GRADE) system as 'very low', 'low', 'moderate' or 'high'.31 32

\section{Statistical methods}

The meta-analysis was performed according to the recommendations of the Cochrane Collaboration using Review Manager (RevMan) (V.5.3) (http://www.cochrane.org). For continuous outcomes and dichotomous outcomes, weighted mean differences (WMDs) and risk ratios (RRs) with their 95\% CIs were reported, respectively. The heterogeneity of meta-analytic pooling was measured using the $\chi^{2}(p<0.1)$ or $I^{2}(<50 \%)$ statistics. A random-effect model by DerSimonian and Laird ${ }^{33}$ was used in all cases. One RCT ${ }^{16}$ with three study arms was included in the meta-analysis, and in order to include each of the two adjunctive PGD arms separately we included the AP monotherapy condition twice in the analysis, but assigned half of the total patients randomised to each PGD arm in order to not inflate the number of AP monotherapy patients, as recommended by prior studies. ${ }^{34}$

Publication bias was assessed using funnel plots and the Egger's test. ${ }^{36}$ All analyses were two-tailed, with alpha set to 0.05 .

\section{RESULTS}

\section{Search results}

Figure 1 presents the flow chart of study selection. Altogether 241 potentially relevant published studies from English (142 trials) and Chinese (99 trials) databases were identified. Of them, three RCTs that compared PGD with BMT, $^{1}$ Guifu Dihuang pill ${ }^{37}$ or biperiden, ${ }^{38}$ respectively, were excluded. Finally, five RCTs ${ }^{1623-26}$ were included in this meta-analysis. 
Table 2 Evaluation of risk of bias in the included studies

\begin{tabular}{|c|c|c|c|c|c|c|c|}
\hline Study & $\begin{array}{l}\text { Random } \\
\text { sequence } \\
\text { generation }\end{array}$ & $\begin{array}{l}\text { Allocation } \\
\text { concealment }\end{array}$ & $\begin{array}{l}\text { Blinding of } \\
\text { participants } \\
\text { and providers }\end{array}$ & $\begin{array}{l}\text { Blinding of } \\
\text { outcome } \\
\text { assessment }\end{array}$ & $\begin{array}{l}\text { Incomplete } \\
\text { outcome data } \\
\text { addressed }\end{array}$ & $\begin{array}{l}\text { Selective } \\
\text { reporting }\end{array}$ & Other biases \\
\hline Xie et al, $2015^{16}$ & Low & High & High & High & Low & Unclear & Unclear \\
\hline Gu et al, $2016^{23}$ & Low & High & High & High & High & Unclear & Unclear \\
\hline $\begin{array}{l}\text { Man et al, } \\
2016^{24}\end{array}$ & Low & Low & Low & Low & Unclear & Low & Unclear \\
\hline Yue, $2016^{26}$ & Low & High & High & High & Low & Unclear & Unclear \\
\hline $\begin{array}{l}\text { Yang et al, } \\
2017^{25}\end{array}$ & Unclear & Unclear & Unclear & Low & Low & Low & Unclear \\
\hline
\end{tabular}

Study characteristics

All five $\mathrm{RCTs}^{1623-26}(\mathrm{n}=450)$ were conducted in China (table 1). These RCTs had a sample size of 90.0 (30.6) (range $=41-120$, median $=99$ ) and an average length of 9.6 (4.1) (range $=4-16$, median=8) weeks. Participants were 30.4 (1.5) (range=28-32, median=30) years old and $80.4 \%$ were female. Only one RCT $^{24}$ mentioned PGD fixed dose $(45.0 \mathrm{~g} /$ day) (table 1$)$.

Quality assessment

Although 80\% (4/5) of the RCTs ${ }^{162324}$ reported randomisation methods with a specific description, only one RCT was rated as low risk regarding allocation concealment (table 2). Similarly, 60\% (3/5) of the RCTs ${ }^{1625} 26$ were rated as low risk in terms of incomplete outcome data. Based on the GRADE approach, the quality of evidence presented for each outcome ranged from 'very low' (14.3\%), 'low' (42.8\%), 'moderate' (14.3\%), to 'high' (28.6\%) (table 3).

\section{Efficacy}

The PGD group had a significant decrease in the serum prolactin level at endpoint compared with the control group (5 RCTs, $\mathrm{n}=380$, WMD: $-32.69 \mathrm{ng} / \mathrm{mL}$ (equivalent to $693.03 \mathrm{mIU} / \mathrm{L})(95 \% \mathrm{CI}-41.66$ to 23.72$), p<0.00001$, $I^{2}=97 \%$; figure 2). Only two $\mathrm{RCTs}^{24} 25$ reported hyperprolactinaemia-induced symptoms. In one RCT, ${ }^{25} 79 \%$

Table 3 GRADE analyses: adjunctive Peony-Glycyrrhiza decoction for antipsychotic-induced hyperprolactinaemia

\begin{tabular}{|c|c|c|c|c|c|c|c|c|}
\hline Outcomes & Subjects & $\begin{array}{l}\text { Risk of } \\
\text { bias }\end{array}$ & Inconsistency & Indirectness & Imprecision & $\begin{array}{l}\text { Publication } \\
\text { bias }\end{array}$ & $\begin{array}{l}\text { Large } \\
\text { effect }\end{array}$ & $\begin{array}{l}\text { Overall quality } \\
\text { of evidence* }\end{array}$ \\
\hline Prolactin level & 380 & No & Serioust & No & No & Serious $\ddagger$ & Large§ & $\begin{array}{l}+/+/+/- \\
\text { Moderate }\end{array}$ \\
\hline $\begin{array}{l}\text { PANSS total } \\
\text { scores }\end{array}$ & 403 & No & No & No & No & No & No & $\begin{array}{l}+/+/+/+ \\
\text { High }\end{array}$ \\
\hline $\begin{array}{l}\text { Discontinuation } \\
\text { due to any } \\
\text { reason }\end{array}$ & 330 & No & No & No & No & No & No & $\begin{array}{l}+/+/+/+ \\
\text { High }\end{array}$ \\
\hline $\begin{array}{l}\text { TESS total } \\
\text { scores }\end{array}$ & 187 & Serious & Serioust & No & No & Serious $\ddagger$ & No & $\begin{array}{l}+/-/-/- \\
\text { Very low }\end{array}$ \\
\hline Akathisia & 190 & Serious & No & No & No & Serious ${ }^{\star *}$ & No & $\begin{array}{l}+/+/-1- \\
\text { Low }\end{array}$ \\
\hline
\end{tabular}

*GRADE Working Group grades of evidence: high quality: further research is very unlikely to change our confidence in the estimate of effect; moderate quality: further research is likely to have an important impact on our confidence in the estimate of effect and may change the estimate; low quality: further research is very likely to have an important impact on our confidence in the estimate of effect and is likely to change the estimate; very low quality: we are very uncertain about the estimate.

†Meta-analytic results presented a serious inconsistency when $l^{2}$ values were greater than $50 \%$ or $p<0.1$ in the $Q$ statistics.

¥For continuous outcomes, $\mathrm{N}<400$.

$\S$ Studies with large effects provided increased quality of evidence. Large effects=effect size $\geq 0.8$.

I|All studies reported as having a serious bias used an open-label method, only mentioned random allocation without describing the method and withdrawal from the study.

${ }^{\star *}$ For dichotomous outcomes, $\mathrm{N}<300$.

GRADE, Grading of Recommendations Assessment, Development and Evaluation; PANSS, Positive and Negative Syndrome Scale; TESS, Treatment Emergent Symptom Scale. 


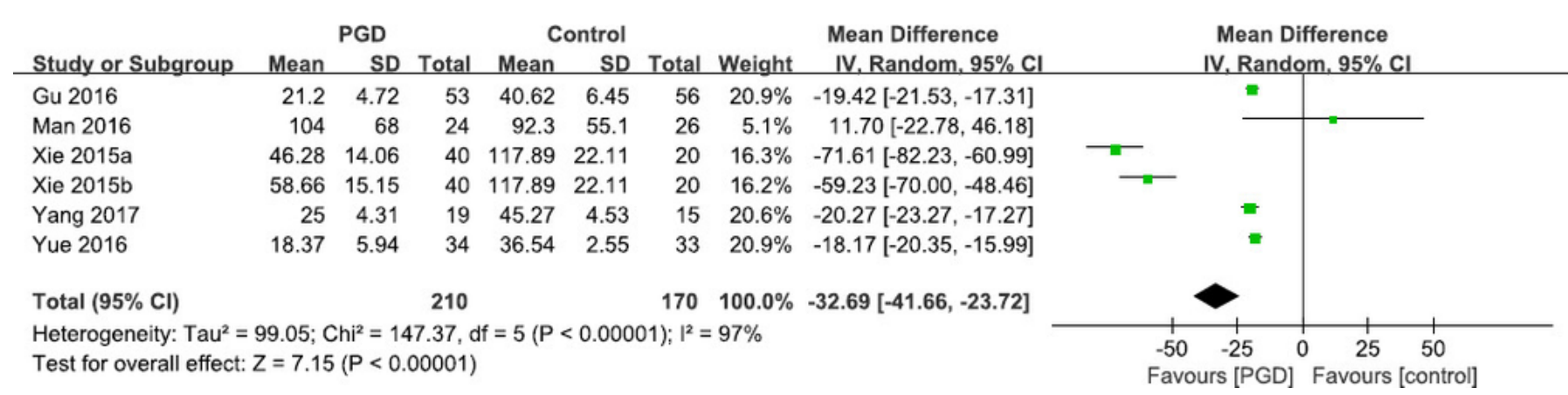

Figure 2 Adjunctive Peony-Glycyrrhiza decoction (PGD) for antipsychotic-induced hyperprolactinaemia: forest plot for serum prolactin level at endpoint $(\mathrm{ng} / \mathrm{mL})$. IV, inverse-variance.

of patients in the PGD group and $0 \%$ in the placebo group reported improvement in hyperprolactinaemia-induced adverse effects. In another RCT, ${ }^{24} 68 \%$ of patients on PGD and $54 \%$ on placebo reported improvement in hyperprolactinaemia-induced adverse effects.

\section{Psychiatric symptoms}

Meta-analysis of PANSS total scores ( 5 RCTs, $n=403$, WMD: $-0.62,95 \%$ CI -2.38 to $1.15, p=0.49, I^{2}=0 \%$ ) showed no significant difference between the PGD augmentation and AP monotherapy groups (table 4).

\section{ADRs and discontinuation rates}

Meta-analysis of akathisia, constipation and headache ( $\mathrm{p}=0.53-0.78$ ) found no significant difference between the PGD group and control group (table 4). Similarly, meta-analysis of TESS total score showed no significant difference (n=187, WMD: $6.95,95 \%$ CI -1.23 to 15.14 , $p=0.10, I^{2}=99 \%$; table 4$)$.

There were similar rates regarding discontinuation due to any reason between the two groups ( 4 RCTs, $n=330$, RR: $0.93,95 \%$ CI 0.63 to $1.37, p=0.71, I^{2}=0 \%$; table 4 ).

\section{Publication bias}

Publication bias could not be evaluated using a funnel plot graph or the Egger's test ${ }^{36}$ since the number of included RCTs was less than $10 .{ }^{39}$

\section{DISCUSSION}

\section{Main findings}

To the best of our knowledge, this was the first meta-analysis examining the efficacy and safety of adjunctive PGD for AP-induced hyperprolactinaemia. The main finding was that adjunctive PGD significantly suppresses elevated prolactin and improved prolactin-induced symptoms, which was consistent with the results of case reports, ${ }^{21}{ }^{22}$ observational studies ${ }^{17-20}$ and a systematic review. ${ }^{40}$ PGD was safe and well tolerated in patients with AP-induced hyperprolactinaemia.

\section{Implications}

There are a few possible reasons for the therapeutic effect of PGD on hyperprolactinaemia. First, PGD may directly suppress the elevated prolactin and testosterone production by affecting their conversion processes and the relevant enzymes. ${ }^{1}$ Second, PGD may modulate the expression of D2 receptor and dopamine transporter. ${ }^{241}$ A recent study ${ }^{42}$ found that paeoniflorin and liquiritin, both major constituents of PGD, are associated with the improvement of hyperprolactinaemia. Third, PGD may inhibit P450 enzymes that metabolise APs. ${ }^{43}$ However, the mechanisms underlying PGD for hyperprolactinaemia need to be further explored.

In clinical practice, the prevalence of AP-induced hyperprolactinaemia has been often underestimated due to lack of visible signs of hyperprolactinaemia-related symptoms, patients' embarrassment with the symptoms and clinicians' lack of awareness. ${ }^{42}$ There are no therapeutic strategies approved by the Food and Drug Administration in the treatment of AP-induced hyperprolactinaemia, although meta-analyses ${ }^{12}{ }^{44}$ have found that adjunctive aripiprazole and metformin may be effective augmentation strategies reducing elevated prolactin

Table 4 Adjunctive Peony-Glycyrrhiza decoction for antipsychotic-induced hyperprolactinaemia: secondary outcomes

\begin{tabular}{|llccc}
\hline Secondary outcomes & Subjects & SMDs/RRs (95\% Cl) & $\mathbf{I}^{\mathbf{2}}$ (\%) & p-value \\
\hline PANSS total scores & 403 & $-0.62(-2.38$ to 1.15$)$ & 0 & 0.49 \\
\hline Discontinuation due to any reason & 330 & $0.93(0.63$ to 1.37$)$ & 0 & 0.71 \\
\hline TESS total scores & 187 & $6.95(-1.23$ to 15.14$)$ & 99 & 0.10 \\
\hline Akathisia & 190 & $1.40(0.46$ to 4.22$)$ & 0 & 0.55 \\
Constipation & 190 & $1.48(0.44$ to 5.02$)$ & 0 & 0.53 \\
Headache & 190 & $0.85(0.27$ to 2.67$)$ & 0 & 0.78 \\
\hline
\end{tabular}

PANSS, Positive and Negative Syndrome Scale;

RRs, risk ratios; SMDs, standard mean differences; TESS, Treatment Emergent Symptom Scale. 
and improving hyperprolactinaemia-induced symptoms. We could not locate any head-to-head trials to compare PGD and aripiprazole/metformin in treating AP-induced hyperprolactinaemia.

Adjunctive PGD appears to be relatively safe and well tolerated. Unlike BMT, a dopamine agonist, adjunctive PGD does not exacerbate psychosis. ${ }^{1}$ Ota ${ }^{38}$ and colleagues have found that adjunctive PGD significantly improved extrapyramidal symptoms.

\section{Strengths and limitations of this study}

The majority strength of this study is the inclusion of Chinese database in the search. The results of this study need to be interpreted with caution due to several limitations. First, only five RCTs met the inclusion criteria and were included in the study. In addition, the sample sizes were relatively small and the methodology of the RCTs was heterogeneous. Second, half of quality of evidence was rated as 'very low' or low' according to the GRADE approach. However, Guyatt ${ }^{45}$ and colleagues suggested that low-quality evidence may still result in strong recommendations as strong recommendations are not always based on high-quality evidence. Third, the dose-response effect of adjunctive PGD in suppressing the elevated prolactin was not analysed due to missing information. Fourth, only English and Chinese databases were searched in this review, while relevant studies may have also been published in other languages.

\section{CONCLUSION}

There has been no effective pharmacotherapy for hyperprolactinaemia, a frequent side effect of AP treatment. Evidence from this meta-analysis supports the use of adjunctive PGD in AP-induced hyperprolactinaemia, which has important clinical implications for the treatment of hyperprolactinaemia. Adjunctive PGD is not associated with increased ADR and exacerbation of psychosis. High-quality RCTs of PGD for hyperprolactinaemia are warranted.

\section{Author affiliations}

${ }^{1}$ Department of Psychiatry, The Affiliated Brain Hospital of Guangzhou Medical University (Guangzhou Huiai Hospital), Guangzhou, China

${ }^{2}$ Department of Neurology, Shenzhen Traditional Chinese Medicine Hospital, Shenzhen, China

${ }^{3}$ Department of Psychiatry, University of Melbourne, Melbourne, Australia

${ }^{4}$ The University of Notre Dame Australia, Fremantle, Australia

${ }^{5}$ Division of Psychiatry, Medical School, University of Western Australia, Perth, Australia

${ }^{6}$ Department of Psychiatry, Mental Health Center of Hebei Province, Baoding, China ${ }^{7}$ Department of Psychiatry, Chongqing Jiangbei Mental Health Hospital, Chongqing, China

${ }^{8}$ Department of Psychiatry, Suzhou Psychiatric Hospital, The Affiliated Guangji Hospital of Soochow University, Suzhou, China

${ }^{9}$ Unit of Psychiatry, Faculty of Health Sciences, University of Macau, Macau, China

Contributors WZ and Y-TX designed the study and were assisted by D-BC in the search for papers, data extraction and analysis. WZ and D-BC drafted the manuscript. GSU, CHN and Y-TX made critical revisions to the manuscript. All authors approved the final version for publication.
Funding The study was supported by the University of Macau (SRG201400019-FHS; MYRG2015-00230 FHS; MYRG2016-00005-FHS) and the Affiliated Brain Hospital of Guangzhou Medical University (2016YFC0906302; 81671334; 2014Y2-00105; 2015BAl13B02). The University of Macau and the Affiliated Brain Hospital of Guangzhou Medical University had no role in the study design, generation or interpretation of the results, and publication of the study.

Competing interests None declared.

Patient consent Not required.

Provenance and peer review Not commissioned; externally peer reviewed.

Data statement All the relevant data has been presented in Tables and Figures.

Open access This is an Open Access article distributed in accordance with the Creative Commons Attribution Non Commercial (CC BY-NC 4.0) license, which permits others to distribute, remix, adapt, build upon this work non-commercially, and license their derivative works on different terms, provided the original work is properly cited and the use is non-commercial. See: http://creativecommons.org/ licenses/by-nc/4.0

\section{REFERENCES}

1 Yuan HN, Wang CY, Sze CW, et al. A randomized, crossover comparison of herbal medicine and bromocriptine against risperidone-induced hyperprolactinemia in patients with schizophrenia. J Clin Psychopharmacol 2008;28:264-370.

2 Wang D, Wang W, Zhou Y, et al. Studies on the regulatory effect of Peony-Glycyrrhiza Decoction on prolactin hyperactivity and underlying mechanism in hyperprolactinemia rat model. Neurosci Lett 2015;606:60-5.

3 Gallego JA, Nielsen J, De Hert M, et al, et al. Safety and tolerability of antipsychotic polypharmacy. Expert Opin Drug Saf 2012;11:527-42.

4 Fitzgerald P, Dinan TG. Prolactin and dopamine: what is the connection? A review article. J Psychopharmacol 2008;22(2 Suppl):12-19.

5 Kim EY, Kim SH, Lee NY, et al. Relationship between prolactin levels and subjective endocrine-related adverse effects in patients with schizophrenia receiving long-term treatment with amisulpride. Pharmacopsychiatry 2012;45:57-63.

6 Berner MM, Hagen M, Kriston L. A systematic review of research on strategies for the management of antipsychotic-induced sexual dysfunction: high-level evidence is needed. J Clin Psychiatry 2006;67:1649-50.

7 Byerly M, Suppes T, Tran QV, et al. Clinical implications of antipsychotic-induced hyperprolactinemia in patients with schizophrenia spectrum or bipolar spectrum disorders: recent developments and current perspectives. J Clin Psychopharmacol 2007;27:639-61.

8 Marken PA, Haykal RF, Fisher JN. Management of psychotropicinduced hyperprolactinemia. Clin Pharm 1992;11:851-6.

9 Bo QJ, Wang ZM, Li XB, et al. Adjunctive metformin for antipsychotic-induced hyperprolactinemia: a systematic review. Psychiatry Res 2016;237:257-63.

10 Bhattacharjee J, El-Sayeh HG. Aripiprazole versus typical antipsychotic drugs for schizophrenia. Cochrane Database Syst Rev 2008;3:CD006617.

11 Kane JM, Correll CU, Goff DC, et al. A multicenter, randomized, double-blind, placebo-controlled, 16-week study of adjunctive aripiprazole for schizophrenia or schizoaffective disorder inadequately treated with quetiapine or risperidone monotherapy. J Clin Psychiatry 2009;70:1348-57.

12 Li X, Tang Y, Wang C. Adjunctive aripiprazole versus placebo for antipsychotic-induced hyperprolactinemia: meta-analysis of randomized controlled trials. PLoS One 2013;8:e70179.

13 Wang CY, Xiang YT, Risperidone Maintenance Treatment in Schizophrenia (RMTS) investigators. Risperidone maintenance treatment in schizophrenia: a randomized, controlled trial. Am J Psychiatry 2010;167:676-85.

14 Gianfrancesco FD, Grogg AL, Mahmoud RA, et al. Differential effects of risperidone, olanzapine, clozapine, and conventional antipsychotics on type 2 diabetes: findings from a large health plan database. J Clin Psychiatry 2002;63:920-30.

15 McQuade RD, Stock E, Marcus R, et al. A comparison of weight change during treatment with olanzapine or aripiprazole: results from a randomized, double-blind study. J Clin Psychiatry 2004;65(Suppl 18):47-56.

16 XIE SS, DING L, CHEN YQ. Effects of peony licorice bolus of risperidone with different proportions on female schizophrenia 
patients suffered hyperprolactinemia [In Chinese]. Medical Research and Education 2015;32:35-8.

17 DUAN D X, CUI GM. The efficacy and safety of shaoyao gancao decoction for treating antipsychotic-induced hyperprolactinemia [In Chinese]. Lishizhen Medicine and Materia Medica Research 2011;22:1471-2.

18 XIANG XM, PAN BB, LI H. Clinical study on shaoyao gancao decoction for treating risperidone-induced hyperprolactinemia [In Chinese]. Medical Innovation of China 2013;10:20-1-2.

19 Yamada K, Kanba S, Murata T, et al. Effectiveness of shakuyakukanzo-to in neuroleptic-induced hyperprolactinemia: a preliminary report. Psychiatry Clin Neurosci 1996;50:341-2.

20 Yamada K, Kanba S, Yagi G, et al. Effectiveness of herbal medicine (shakuyaku-kanzo-to) for neuroleptic-induced hyperprolactinemia. J Clin Psychopharmacol 1997:17:234-5.

21 Hori H, Yoshimura R, Katsuki A, et al. Herbal medicine (Shakuyakukanzo-to) improves olanzapine-associated hyperprolactinemia: a case report. J Clin Psychopharmacol 2013;33:122-3.

22 Yamada K, Kanba S, Yagi G, et al. Herbal medicine (Shakuyakukanzo-to) in the treatment of risperidone-induced amenorrhea. J Clin Psychopharmacol 1999;19:380-1.

23 GU P, GE X, LI X. Treatment of olanzapine-induced hyperprolactinemia by Shaoyao Gancao Decoction [In Chinese]. Chinese Journal of Integrated Traditional and Western Medicine 2016;36:1456-9.

24 Man SC, Li XB, Wang HH, et al. Peony-glycyrrhiza decoction for antipsychotic-related hyperprolactinemia in women with schizophrenia: a randomized controlled trial. J Clin Psychopharmaco 2016;36:572-9.

25 Yang P, Li L, Yang D, et al. Effect of peony-glycyrrhiza decoction on amisulpride-induced hyperprolactinemia in women with schizophrenia: a preliminary study. Evid Based Complement Alternat Med 2017;2017:1-8.

26 YUE LF. The effection of Shaoyao Gancao Decoction in treating hyperprolactinemia caused by antipsychotics and cognitive function [In Chinese] [D: XinXiang Medical University, 2016.

27 Kay SR, Fiszbein A, Opler LA. The positive and negative syndrome scale (PANSS) for schizophrenia. Schizophr Bull 1987;13:261-76.

28 Overall JE, Gorham DR. The brief psychiatric rating scale. Psychol Rep 1962;10:799-812.

29 GUY W. ECDEU assessment manual for psychopharmacology (Revised) [M]. Bethesda, MD: US Department of Health, Education, and Welfare, 1976

30 HIGGINS J, Green S. 2011.Cochrane handbook for systematic reviews of interventions version 5.1.0 [updated March 2011]. The Cochrane Collaboration. www.cochrane-handbook.org.

31 Balshem H, Helfand M, Schünemann $\mathrm{HJ}$, et al. GRADE guidelines: 3. Rating the quality of evidence. J Clin Epidemiol 2011;64:401-6.
32 Atkins D, Best D, GRADE Working Group. Grading quality of evidence and strength of recommendations. BMJ 2004;328:1490.

33 DerSimonian R, Laird N. Meta-analysis in clinical trials. Control Clin Trials 1986;7:177-88.

$34 \mathrm{Gu} \mathrm{XJ}$, Chen $\mathrm{R}$, Sun $\mathrm{CH}$, et al. Effect of adjunctive ranitidine for antipsychotic-induced weight gain: a systematic review of randomized placebo-controlled trials. J Int Med Res 2018;46:22-32.

35 Galling B, Roldán A, Hagi K, et al. Antipsychotic augmentation vs. monotherapy in schizophrenia: systematic review, meta-analysis and meta-regression analysis. World Psychiatry 2017;16:77-89.

36 Egger M, Davey Smith G, Schneider M, et al. Bias in meta-analysis detected by a simple, graphical test. BMJ 1997;315:629-34.

37 PAN YY, LI W, CHEN W. A comparative study of shaoyao gancao decoction and Guifu Dihuang pill in the treatment of risperidone induced hyperprolactinemia [In Chinese]. Modern Journal of integrated traditional Chinese and Western Medicine 2016;5:3947-9.

38 Ota T, Miura I, Kanno-Nozaki K, et al. Effects of ShakuyakuKanzo-to on extrapyramidal symptoms during antipsychotic treatment: a randomized, open-label study. J Clin Psychopharmacol 2015;35:304-7.

39 Sterne JA, Sutton AJ, loannidis JP, et al. Recommendations for examining and interpreting funnel plot asymmetry in meta-analyses of randomised controlled trials. BMJ 2011:343:d4002.

40 HASAM-RANJBAR S, VAHIDI H, TASLIMI S. A systematic review on the efficacy of herbal medicines in the management of human druginduced hyperprolactinemia; potential sources for the development of novel drugs. Int $J$ Pharmacol 2010;6:691-5.

41 Wang D, Wong HK, Zhang L, et al. Not only dopamine D2 receptors involved in Peony-Glycyrrhiza Decoction, an herbal preparation against antipsychotic-associated hyperprolactinemia. Prog Neuropsychopharmacol Biol Psychiatry 2012;39:332-8.

42 Chen FY. The Research of the Treatment for Mastopathia by LiverSpleen-Harmonizing Therapy of Jinkui Yaolue-Experimental Study on Effect of Dangguishaoyao Modified Decoction on LH,FSH,PRL and Histomorphology in Rats [D: Fujian College of Traditional Chinese Medicine, 2008.

43 Wang W, Tian DD, Zheng B, et al. Peony-Glycyrrhiza Decoction, an Herbal Preparation, Inhibits Clozapine Metabolism via Cytochrome P450s, but Not Flavin-Containing Monooxygenase in In Vitro Models. Drug Metab Dispos 2015;43:1147-53.

44 Zheng W, Yang XH, Cai DB, et al. Adjunctive metformin for antipsychotic-related hyperprolactinemia: A meta-analysis of randomized controlled trials. J Psychopharmacol 2017;31:625-31.

45 Guyatt GH, Oxman AD, GRADE Working Group. GRADE: an emerging consensus on rating quality of evidence and strength of recommendations. BMJ 2008;336:924-6.

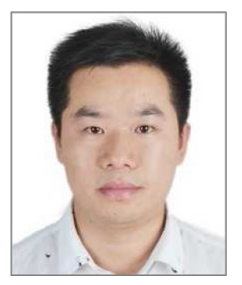

Dr Wei Zheng obtained a bachelor's degree from Hebei Medical University in 2012 and a master's degree in psychiatry and mental health from the Capital Medical University in Beijing in 2015. Since then he has been working as a resident physician in the Department of Psychiatry in the Guangzhou Huiai Hospital. He is currently a PhD Candidate at the Affiliated Brain Hospital of Guangzhou Medical University (Guangzhou Huiai Hospital). His main research interest is depression and schizophrenia.

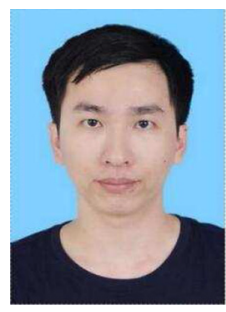

Dr Dong-bin Cai obtained a bachelor's degree from Guangzhou University of Chinese Medicine in 2015 and a master's degree in clinical foundation of Chinese Medicine from Guangzhou University of Chinese Medicine in 2018. His main research interest is depression and schizophrenia 